\title{
STABILITY PROPERTIES OF THE CLASS OF ASYMPTOTIC MARTINGALES
}

\author{
BY ALEXANDRA BELLOW 1
}

Communicated by Alexandra Bellow, December 18, 1975

1. Let $(\Omega, F, P)$ be a probability space. Let $N=\{1,2,3, \ldots\}$ and let $\left(F_{n}\right)_{n \in N}$ be an increasing sequence of sub- $\sigma$-algebras of $F$, i.e. if $n \leqslant m$ then $F_{n}$ $\subset F_{n}$. A bounded stopping time (with respect to the sequence $\left.\left(F_{n}\right)_{n \in N}\right)$ is a mapping $\tau: \Omega \rightarrow N$ such that $\{\tau=n\} \in F_{n}$ for all $n \in N$ and $\tau$ assumes only finitely many values. Let $T$ be the set of all bounded stopping times. With the definition $\tau \leqslant \sigma$ if $\tau(\omega) \leqslant \sigma(\omega)$ for all $\omega \in \Omega, T$ is a directed set "filtering to the right" (note that if $\tau \in T, \sigma \in T$, then $\tau \vee \sigma \in T, \tau \wedge \sigma \in T$ ). For $\tau \in T$ recall that $F_{\tau}=\left\{A \in F \mid A \cap\{\tau=n\} \in F_{n}\right.$ for all $\left.n \in N\right\}$ and that $\tau \leqslant \sigma \mathrm{im}$. plies $F_{\tau} \subset F_{\sigma}$.

Let $E$ be a Banach space. Let $X_{n}: \Omega \rightarrow E$ for each $n \in N$. The sequence $\left(X_{n}\right)_{n \in N}$ is called adapted if $X_{n}: \Omega \rightarrow E$ is Bochner $F_{n}$-measurable for each $n \in N$

The notion of asymptotic martingale has received a great deal of attention in the last few years; it provides a unified and elegant treatment for martingales, submartingales, supermartingales, quasimartingales $[1],[2],[5]$. We recall its definition:

Definition. An adapted sequence $\left(X_{n}\right)_{n \in N}$ of E-valued random variables is called an E-valued asymptotic martingale if $X_{n}$ is Bochner integrable, i.e. $\int\left\|X_{n}(\omega)\right\| d P(\omega)<\infty$ for all $n \in N$ and $\left(\int X_{\tau}\right)_{\tau \in T}$ converges in the norm topology of $E$.

We recall the fundamental a.e. convergence theorems for asymptotic martingales:

(I) Let $\left(X_{n}\right)_{n \in N}$ be a real-valued asymptotic martingale. Suppose that $\sup _{n \in N} \int\left|X_{n}\right|<\infty$. Then $\left(X_{n}\right)_{n \in N}$ converges to a limit a.e. (see [1]).

(II) Let $\left(X_{n}\right)_{n \in N}$ be an $E$-valued asymptotic martingale. Suppose that $\sup _{\tau \in T} \int\left\|X_{\tau}\right\|<\infty$. Then even under the best circumstances (if $E$ is Hilbert space $\left.l^{2}\right)$ the sequence $\left(X_{n}\right)_{n \in N}$ need not converge in the norm topology of $E$, but only weakly a.e. (see [2]).

Nevertheless the following is true without any restriction on the Banach space $E$ (see also [2], Lemma 2):

AMS (MOS) subject classifications (1970). Primary 60G40, 60G45; Secondary 28A65, 28A20.

${ }^{1}$ The main results of this note were announced in a Colloquium lecture given at the Hebrew University of Jerusalem, on November 27, 1975. 
THEOREM 1. Let $E$ be a Banach space. Let $\left(X_{n}\right)_{n \in N}$ be an E-valued asymptotic martingale. For each $\tau \in T$ let $\mu_{\tau}(A)=\int_{A} X_{\tau}$, for $A \in F_{\tau}$. Then:

(1) The family $\left(\mu_{\tau}(A)\right)_{\tau}$ converges to a limit, $\mu(A)$, for each $A \in F_{\infty}=$ $\bigcup_{\tau \in T} F_{\tau}=\bigcup_{n \in N} F_{n}$, and the convergence is "uniform" on $F_{\infty}$, that is for each $\epsilon>0$ there is $\tau_{0} \in T$ such that

$$
\sigma \in T, \sigma \geqslant \tau_{0} \Rightarrow\left\|\mu_{\sigma}(A)-\mu(A)\right\| \leqslant \epsilon \quad \text { for all } A \in F_{\sigma} .
$$

(2) Furthermore if $\sup _{n \in N} \int\left\|X_{n}\right\|<\infty$, then there is $M>0$ such that $\left\|\mu_{\tau}(A)\right\| \leqslant M$ for each $\tau \in T$ and $A \in F_{\tau}$.

2. In the Lemma that follows we assume that: $\Omega$ is a set, $A$ a Boolean algebra of subsets of $\Omega, T$ a directed set filtering to the right for " $\leqslant$ ", and $\left(A_{t}\right)_{t \in T}$ an increasing family of sub-algebras of $A$, that is: $s \leqslant t \Rightarrow A_{s} \subset A_{t}$.

For any real-valued bounded additive set function $v$ defined on a Boolean algebra of subsets of $\Omega$, we write $v=v^{+}-v^{-}$for the Jordan decomposition of $v$ (see [4, pp. 98-99]).

The following Lemma may be regarded as a variant of E. H. Moore's double limit lemma (see $[4$, p. 28]):

Lemma. For each $t \in T$ let $\mu_{t}: A_{t} \rightarrow R$ be an additive set function. We assume that:

(i) There is $M>0$ such that $\left|\mu_{t}(A)\right| \leqslant M$ for each $t \in T$ and $A \in A_{t}$.

(ii) The family $\left(\mu_{t}(A)\right)_{t}$ converges to a limit, $\mu(A)$, for each $A \in A_{\infty}=$ $\bigcup_{t \in T} A_{t}$, and the convergence is "uniform" on $A_{\infty}$, that is for each $\epsilon>0$ there is $t_{0} \in T$ such that

$$
s \in T, s \geqslant t_{0} \Rightarrow\left|\mu_{s}(A)-\mu(A)\right| \leqslant \epsilon \text { for all } A \in \mathrm{A}_{s} .
$$

Then $\lim _{t \in T} \mu_{t}^{+}(\Omega)$ and $\lim _{t \in T} \mu_{t}^{-}(\Omega)$ exist and equal $\mu^{+}(\Omega)$ and $\mu^{-}(\Omega)$, respectively.

With the notation of $\S 1$, the following result, first proved in [1] (see also [5]), is an easy consequence of Theorem 1 and the previous Lemma:

Corollary. Let $\left(X_{n}\right)_{n \in N}$ be a real-valued asymptotic martingale and suppose that $\sup _{n \in N} \int\left|X_{n}\right|<\infty$. Then $\left(X_{n}^{+}\right)_{n \in N},\left(X_{n}^{-}\right)_{n \in N}$ are asymptotic martingales.

Before stating the next theorem we note that the class of continuous functions $\Phi: R \rightarrow R$ for which $\lim _{x \rightarrow+\infty}(\Phi(x) / x), \lim _{x \rightarrow-\infty}(\Phi(x) / x)$ exist (finite or infinite) is quite large: it includes the piecewise linear functions, the convex functions, the concave function, the subadditive functions.

THEOREM 2. Let $\Phi: R \rightarrow R$ be continuous and such that $\lim _{x \rightarrow+\infty}(\Phi(x) / x)$ and $\lim _{x \rightarrow-\infty}(\Phi(x) / x)$ exist and are finite. Let $\left(X_{n}\right)_{n \in N}$ be any real-valued asymp- 
totic martingale such that $\sup _{n \in N} \int\left|X_{n}\right|<\infty$. Then $\left(\Phi\left(X_{n}\right)\right)_{n \in N}$ is an asymptotic martingale and $\sup _{n \in N} \int\left|\Phi\left(X_{n}\right)\right|<\infty$.

3. For simplicity we assume in this section that $\Omega=[0,1], F=$ the $\sigma$ algebra of Borel sets and $P$ a nonatomic probability measure. When the sequence of $\sigma$-algebras is not explicitly mentioned, it is assumed that $\left(F_{n}\right)_{n \in N}$ is the "minimal", sequence, that is $F_{n}=\sigma\left(X_{1}, X_{2}, \ldots, X_{n}\right)$ for each $n \in N$.

In a certain sense Theorem 2 is best possible, as the following result shows:

Theorem 3. Let $\Phi: R \rightarrow R$ be continuous and such that $\lim _{x \rightarrow+\infty}(\Phi(x) / x)$ $=+\infty$. Then there is a real-valued asymptotic martingale $\left(X_{n}\right)_{n \in N}$ such that $\sup _{n \in N} \int\left|X_{n}\right|<\infty, \sup _{n \in N} \int\left|\Phi\left(X_{n}\right)\right|<\infty$, but $\left(\Phi\left(X_{n}\right)\right)_{n \in N}$ is not an asymptotic martingale.

REMARK. The standard examples of functions $\Phi: R \rightarrow R$ satisfying $\lim _{x \rightarrow+\infty}(\Phi(x) / x)=+\infty$ are $|x| \log ^{+}|x|$ and $|x|^{p} \quad(p>1)$. The classical theorems from martingale theory concerning these functions [3, pp. 295-296] do not not carry over to asymptotic martingales, as Theorem 3 shows.

THEOREM 4. Let $S: \Omega \rightarrow \Omega$ be an ergodic measure-preserving transformation. There are then functions $f \in L_{+}^{1}$ such that if we set

$$
X_{n}=\left(f+f \circ S+\cdots+f \circ S^{n-1}\right) / n \text {, }
$$

for each $n \in N$, then $\left(X_{n}\right)_{n \in N}$ is not an asymptotic martingale.

To conclude: the notion of asymptotic martingale is an important and useful concept. Nevertheless it has its limitations, as Theorems 3 and 4 above show.

\section{REFERENCES}

1. D. G. Austin, G. A. Edgar and A. Ionescu Tulcea, Pointwise convergence in terms of expectations, Z. Wahrscheinlichkeitstheorie und Verw. Gebiete 30 (1974), 17-26.

2. R. V. Chacon and L. Sucheston, On convergence of vector-valued asymptotic martingales, Z. Wahrscheinlichkeitstheorie und Verw. Gebiete 33 (1975), 55-59.

3. J. L. Doob, Stochastic processes, Wiley, New York; Chapman \& Hall, London, 1953. MR 15, 445 .

4. N. Dunford and J. T. Schwartz, Linear operators. I: General theory, Pure and Appl. Math., vol. 7, Interscience, New York, 1958. MR 22 \#3302.

5. G. A. Edgar and L. Sucheston, Amarts, a class of asymptotic martingales (Discrete parameters), J. Multivariate Anal. (to appear).

DEPARTMENT OF MATHEMATICS, NORTHWESTERN UNIVERSITY, EVANSTON, ILLINOIS 60201 